\begin{tabular}{l} 
Universal Journal of Mathematics and Applications, 4(3) (2021) 107-113 \\
Research paper \\
Universal Journal of Mathematics and Applications \\
Journal Homepage: www.dergipark.gov.tr/ujma \\
ISSN 2619-9653 \\
DOI: https://doi.org/10.32323/ujma.937479 \\
\hline
\end{tabular}

\title{
A Study on $f$-Rectifying Curves in Euclidean $n$-Space
}

\author{
Zafar Iqbal $^{1 *}$ and Joydeep Sengupta ${ }^{2}$ \\ ${ }^{1}$ Department of Mathematics, Kaliyaganj College, Uttar Dinajpur - 733129, West Bengal, India \\ ${ }^{2}$ Department of Mathematics and Statistics, Aliah University, Kolkata - 700160, West Bengal, India \\ ${ }^{*}$ Corresponding author
}

\author{
Article Info \\ Keywords: Euclidean space, Frenet- \\ Serret formulae, Higher curvatures, \\ Rectifying curve, $f$-position vector field, \\ $f$-rectifying curve. \\ 2010 AMS: 53A04, 53B25, 53C40. \\ Received: 15 May 2021 \\ Accepted: 1 October 2021 \\ Available online: 1 October 2021
}

\begin{abstract}
A rectifying curve in Euclidean $n$-space $\mathbb{E}^{n}$ is defined as an arc-length parametrized curve $\gamma$ in $\mathbb{E}^{n}$ such that its position vector always lies in its rectifying space (i.e., the orthogonal complement of its principal normal vector field) in $\mathbb{E}^{n}$. In this paper, in analogy to this, we introduce the notion of an $f$-rectifying curve in $\mathbb{E}^{n}$ as a curve $\gamma$ in $\mathbb{E}^{n}$ parametrized by its arc-length $s$ such that its $f$-position vector field $\gamma_{f}$, defined by $\gamma_{f}(s)=\int f(s) d \gamma$, always lies in its rectifying space in $\mathbb{E}^{n}$, where $f$ is a nowhere vanishing real-valued integrable function in parameter $s$. The main purpose is to characterize and classify such curves in $\mathbb{E}^{n}$.
\end{abstract}

\section{Introduction}

Let $\mathbb{E}^{3}$ denote the Euclidean 3-space (i.e., the three-dimensional real vector space $\mathbb{R}^{3}$ endowed with the standard inner product $\langle\cdot, \cdot\rangle$ ). Let $\gamma: I \longrightarrow \mathbb{E}^{3}$ be a unit-speed curve (i.e., a curve in $\mathbb{E}^{3}$ parametrized by arc length function $s$ ) of class at least $\mathscr{C}^{3}$ (i.e., possessing continuous derivatives at least up to third order). Needless to mention, $I$ denotes a non-trivial interval in $\mathbb{R}$, i.e., a connected set in $\mathbb{R}$ containing at least two points. We consider the Frenet apparatus $\left\{T_{\gamma}, N_{\gamma}, B_{\gamma}, \kappa_{\gamma}, \tau_{\gamma}\right\}$ for the curve $\gamma$ which is defined as follows: $T_{\gamma}=\gamma^{\prime}$ is the unit tangent vector field along $\gamma ; N_{\gamma}$ is the unit principal normal vector field along $\gamma$ obtained by normalizing the acceleration vector field $T_{\gamma}^{\prime}$; $B_{\gamma}=T_{\gamma} \times N_{\gamma}$ is the unit binormal vector field along $\gamma$ and it is the unique vector field along $\gamma$ orthogonal to both $T_{\gamma}$ and $N_{\gamma}$ so that the dynamic Frenet frame $\left\{T_{\gamma}, N_{\gamma}, B_{\gamma}\right\}$ is positive definite along $\gamma$ having the same orientation as that of $\mathbb{E}^{3} ; \kappa_{\gamma}$ is the curvature and $\tau_{\gamma}$ is the torsion of $\gamma$. If $\gamma$ is of class at least $\mathscr{C}^{5}$, then its curvature $\kappa_{\gamma}$ and torsion $\tau_{\gamma}$ are at least twice differentiable. Moreover, $\gamma$ reduces to a tortuous curve in $\mathbb{E}^{3}$ if it has nowhere vanishing curvature $\kappa_{\gamma}$ and torsion $\tau_{\gamma}$ (cf. [1] or [2]).

At each point $\gamma(s)$ on $\gamma$, the planes spanned by $\left\{T_{\gamma}(s), N_{\gamma}(s)\right\},\left\{T_{\gamma}(s), B_{\gamma}(s)\right\}$ and $\left\{N_{\gamma}(s), B_{\gamma}(s)\right\}$ are respectively called the osculating plane, rectifying plane and normal plane of $\gamma([1,2])$. It is well known from elementary Differential Geometry that a space curve $\gamma$ lies in a plane in $\mathbb{E}^{3}$ if its position vector field always lies in its osculating planes, and it lies on a sphere in $\mathbb{E}^{3}$ if its position vector field always lies in its normal planes. In this point of view, it is natural to inquire the geometric question: Does there exist a space curve $\gamma: I \longrightarrow \mathbb{E}^{3}$ whose position vector field always lies in its rectifying planes? The existence of such space curves was introduced by B.Y. Chen in his paper [3] and named as rectifying curves. Thus, the position vector field of a rectifying curve $\gamma: I \longrightarrow \mathbb{E}^{3}$ parametrized by arc length function $s$ satisfies the equation

$$
\gamma(s)=\lambda(s) T_{\gamma}(s)+\mu(s) B_{\gamma}(s)
$$

for some smooth functions $\lambda, \mu: I \longrightarrow \mathbb{R}$. In [3], B.Y. Chen explored some characterizations of rectifying curves in $\mathbb{E}^{3}$ in terms of distance functions, tangential, normal and binormal components of their position vector field and also in terms of ratios of their curvature and torsion. Also, he attempted for a classification of such curves in $\mathbb{E}^{3}$ based on a sort of dilation applied on unit-speed curves on the unit sphere $\mathbb{S}^{2}(1)$.

In [4], B.Y. Chen and F. Dillen observed that rectifying curves can be viewed as centrodes and extremal curves in $\mathbb{E}^{3}$. Moreover, they found a relation between rectifying curves and centrodes which performs a significant role in defining the curves of constant procession in Differential Geometry as well as in Kinematics or, in general, Mechanics. Thereafter, several characterizations of rectifying curves in 
Euclidean spaces were evolved in [5-8]. Meanwhile, the notion of rectifying curves were extended to several ambient spaces, e.g., 3D sphere $\mathbb{S}^{3}(r)$ [9], 3D hyperbolic space $\mathbb{H}^{3}(-r)$ [10], Minkowski 3-space $\mathbb{E}_{1}^{3}[11,12]$, Minkowski space-time $\mathbb{E}_{1}^{4}$ [13-15]. Furthermore, a new kind of curves were studied in $\mathbb{E}^{3}$ which generalizes rectifying curves and helices [16]. Also, some characterizations and classification of non-null and null $f$-rectifying curves (which are a sort of generalization of rectifying curves) were investigated in Minkowski 3-space $\mathbb{E}_{1}^{3}[17,18]$, Minkowski space-time $\mathbb{E}_{1}^{4}[19]$ and Euclidean 4-space [20].

In section 2, we give requisite preliminaries and then, in section 3, we introduce the notion of $f$-rectifying curves in $\mathbb{E}^{n}$. Thereafter, section 4 is devoted to investigate some simple geometric characterizations of $f$-rectifying curves in $\mathbb{E}^{n}$. Afterwards, section 5 is dedicated to classify $f$-rectifying curves in terms of their $f$-position vectors in $\mathbb{E}^{n}$. Finally, we conclude our study in section 6 . This is how the paper is organised.

\section{Preliminaries}

The Euclidean $n$-space $\mathbb{E}^{n}$ is the $n$-dimensional real vector space $\mathbb{R}^{n}$ equipped with the standard inner product $\langle\cdot, \cdot\rangle$ defined by

$$
\langle x, y\rangle:=\sum_{i=1}^{n} x_{i} y_{i}
$$

for all tangent vectors $x=\left(x_{1}, x_{2}, \ldots, x_{n}\right), y=\left(y_{1}, y_{2}, \ldots, y_{n}\right)$ to $\mathbb{R}^{n}$. As usual, the norm or length of a tangent vector $x=\left(x_{1}, x_{2}, \ldots, x_{n}\right)$ to $\mathbb{R}^{n}$ is denoted and defined by

$$
\|x\|:=\sqrt{\langle x, x\rangle}=\sqrt{\sum_{i=1}^{n} x_{i}^{2}} .
$$

Let $\gamma: J \longrightarrow \mathbb{E}^{n}$ be an arbitrary differentiable curve parametrized by $t$ and $\gamma^{\prime}$ denotes its velocity vector field in $\mathbb{E}^{n}$. Also, we assume that $\gamma$ is regular, i.e., its velocity vector field $\gamma^{\prime}$ is nowhere vanishing. If we change the parameter $t$ by arc-length function $s: J \longrightarrow I$ based at $t_{0}$ given by

$$
s(t)=\int_{t_{0}}^{t}\left\|\gamma^{\prime}(u)\right\| d u
$$

such that $\left\|\gamma^{\prime}(s)\right\|=\sqrt{\left\langle\gamma^{\prime}(s), \gamma^{\prime}(s)\right\rangle}=1$, i.e., $\left\langle\gamma^{\prime}(s), \gamma^{\prime}(s)\right\rangle=1$, then $\gamma: I \longrightarrow \mathbb{E}^{n}$ is referred to as an arc-length parametrized or a unit-speed curve in $\mathbb{E}^{n}$. We may consider that $\gamma$ is of class at least $\mathscr{C}^{4}$. Now, let $T_{\gamma}, N_{\gamma}$ denote respectively the unit tangent vector field and the unit principal normal vector field of $\gamma$ and for each $i \in\{1,2, \ldots, n-2\}$, let $B_{\gamma_{i}}$ denote the unit $i$-th binormal vector field of $\gamma$ so that $\left\{T_{\gamma}, N_{\gamma}, B_{\gamma_{1}}, B_{\gamma_{2}}, \ldots, B \gamma_{n-2}\right\}$ forms the positive definite dynamic Frenet frame along $\gamma$ having the same orientation as that of $\mathbb{E}^{n}$. Also, for each $i \in\{1,2, \ldots, n-1\}$, let $\kappa_{\gamma_{i}}$ denote the $i$-th curvature of $\gamma$. Then the Frenet-Serret formulae for the curve $\gamma$ are given by ( [21,22])

$$
\left(\begin{array}{c}
T_{\gamma}^{\prime} \\
N_{\gamma}^{\prime} \\
B \gamma_{1}^{\prime} \\
B \gamma_{2}^{\prime} \\
\vdots \\
B \gamma_{n-2}^{\prime}
\end{array}\right)=\left(\begin{array}{ccccccc}
0 & \kappa_{\gamma_{1}} & 0 & 0 & \cdots & 0 & 0 \\
-\kappa_{\gamma_{1}} & 0 & \kappa_{\gamma_{2}} & 0 & \cdots & 0 & 0 \\
0 & -\kappa_{\gamma_{2}} & 0 & \kappa_{\gamma_{3}} & \cdots & 0 & 0 \\
0 & 0 & -\kappa_{\gamma_{3}} & 0 & \cdots & 0 & 0 \\
\vdots & \vdots & \vdots & \vdots & \ddots & \vdots & \vdots \\
0 & 0 & 0 & 0 & \cdots & \kappa_{\gamma_{n-1}} & 0
\end{array}\right)\left(\begin{array}{c}
T_{\gamma} \\
N_{\gamma} \\
B_{\gamma_{1}} \\
B_{\gamma_{2}} \\
\vdots \\
B_{\gamma_{n-2}}
\end{array}\right) .
$$

From the above formulae, it follows that $\kappa_{\gamma_{n-1}} \not \equiv 0$ if and only if the curve $\gamma$ lies wholly in $\mathbb{E}^{n}$. This is equivalent to saying that $\kappa_{\gamma_{n-1}} \equiv 0$ if and only if the curve $\gamma$ lies wholly in a hypersurface in $\mathbb{E}^{n}$ (cf. $[21,22]$ ). We recall that the hypersurface in $\mathbb{E}^{n}$ defined by

$$
\mathbb{S}^{n-1}(1):=\left\{x \in \mathbb{E}^{n}:\langle x, x\rangle=1\right\}
$$

is called the unit sphere with centre at the origin in $\mathbb{E}^{n}$. We also recall that the rectifying space of the curve $\gamma$ in $\mathbb{E}^{n}$ is the orthogonal complement $N_{\gamma}{ }^{\perp}$ of its principal normal vector field $N_{\gamma}$ in $\mathbb{E}^{n}$ defined by

$$
N_{\gamma}^{\perp}:=\left\{x \in \mathbb{E}^{n}:\left\langle x, N_{\gamma}\right\rangle=0\right\} .
$$

\section{Notion of $f$-rectifying curves in $\mathbb{E}^{n}$}

Let $\gamma: I \longrightarrow \mathbb{E}^{n}$ be a unit-speed curve (parametrized by arc length $s$ ) with Frenet apparatus $\left\{T_{\gamma}, N_{\gamma}, B_{\gamma_{1}}, B_{\gamma_{2}}, \ldots, B_{\gamma_{n-2}}, \kappa_{\gamma_{1}}, \kappa_{\gamma_{2}}, \ldots, \kappa_{\gamma_{n-1}}\right\}$. As found in [8], $\gamma$ is a rectifying curve in $\mathbb{E}^{n}$ if and only if its position vector field always lies in its rectifying space, i.e., if and only if its position vector field satisfies

$$
\gamma(s)=\lambda(s) T_{\gamma}(s)+\sum_{i=1}^{n-2} \mu_{i}(s) B_{\gamma_{i}}(s)
$$

for some differentiable functions $\lambda, \mu_{1}, \mu_{2}, \ldots, \mu_{n-2}: I \longrightarrow \mathbb{R}$. Now, let $f: I \longrightarrow \mathbb{R}$ be a nowhere vanishing integrable function. Then the $f$-position vector field of $\gamma$ is denoted by $\gamma_{f}$ and is defined by

$$
\gamma_{f}(s)=\int f(s) d \gamma
$$

Here, the integral sign is used in this sense that on differentiation of previous equation, one finds

$$
\gamma_{f}^{\prime}(s)=f(s) T_{\gamma}(s)
$$

so that $\gamma_{f}$ is an integral curve of the vector field $f T_{\gamma}$ along $\gamma$ in $\mathbb{E}^{n}$. Using this concept of $f$-position vector field of a curve in $\mathbb{E}^{n}$, we define an $f$-rectifying curve in $\mathbb{E}^{n}$ as follows: 
Definition 3.1. Let $\gamma: I \longrightarrow \mathbb{E}^{n}$ be a unit-speed curve with Frenet apparatus $\left\{T_{\gamma}, N_{\gamma}, B_{\gamma_{1}}, B_{\gamma_{2}}, \ldots, B_{\gamma_{n-2}}, \kappa_{\gamma_{1}}, \kappa_{\gamma_{2}}, \ldots, \kappa_{\gamma_{n-1}}\right\}$ and $f: I \longrightarrow$ $\mathbb{R}$ be a nowhere vanishing integrable function in arc-length parameter s of $\gamma$ with at least $(n-2)$-times differentiable primitive function $F$. Then $\gamma$ is referred to as an $f$-rectifying curve in $\mathbb{E}^{n}$ if its $f$-position vector field $\gamma_{f}$ always lies in its rectifying space in $\mathbb{E}^{n}$, i.e., if its $f$-position vector field $\gamma_{f}$ satisfies the equation

$$
\gamma_{f}(s)=\lambda(s) T_{\gamma}(s)+\sum_{i=1}^{n-2} \mu_{i}(s) B_{\gamma_{i}}(s)
$$

for some differentiable functions $\lambda, \mu_{1}, \mu_{2}, \ldots, \mu_{n-2}: I \longrightarrow \mathbb{R}$.

Remark 3.2. In particular, if the function $f$ is a non-zero constant on $I$, then, up to isometries (rigid motions) of $\mathbb{E}^{n}$, an $f$-rectifying curve $\gamma: I \longrightarrow \mathbb{E}^{n}$ is congruent to a rectifying curve in $\mathbb{E}^{n}$ and the study coincides with the same incorporated in [8].

\section{Some geometric characterizations of $f$-rectifying curves in $\mathbb{E}^{n}$}

In this section, we present some geometrical characterizations of unit-speed $f$-rectifying curves in $\mathbb{E}^{n}$ in terms of the norm functions, tangential components, normal components, binormal components of their $f$-position vector field.

Theorem 4.1. Let $\gamma: I \longrightarrow \mathbb{E}^{n}$ be a unit-speed curve (parametrized by arc length s) having nowhere vanishing $n-1$ curvatures $\kappa_{\gamma_{1}}, \kappa_{\gamma_{2}}, \ldots, \kappa_{\gamma_{n-1}}$ and let $f: I \longrightarrow \mathbb{R}$ be a nowhere vanishing integrable function with at least $(n-2)$-times differentiable primitive function $F$. If $\gamma$ is a $f$-rectifying curve in $\mathbb{E}^{n}$, then the following statements are true:

1. The norm function $\rho=\left\|\gamma_{f}\right\|$ is given by $\rho(s)=\sqrt{F^{2}(s)+c^{2}}$, where $c$ is a non-zero constant.

2. The tangential component $\left\langle\gamma_{f}, T_{\gamma}\right\rangle$ of $\gamma_{f}$ is given by $\left\langle\gamma_{f}(s), T_{\gamma}(s)\right\rangle=F(s)$.

3. The normal component $\gamma_{f}^{N_{\gamma}}$ of $\gamma_{f}$ has a constant length and the norm function $\rho$ is non-constant.

4. The first binormal component $\left\langle\gamma_{f}, B_{\gamma_{1}}\right\rangle$ and the second binormal component $\left\langle\gamma_{f}, B_{\gamma_{2}}\right\rangle$ of $\gamma_{f}$ are respectively given by

$$
\left\langle\gamma_{f}(s), B_{\gamma_{1}}(s)\right\rangle=\frac{\kappa_{\gamma_{1}}(s)}{\kappa_{\gamma_{2}}(s)} F(s), \quad\left\langle\gamma_{f}(s), B_{\gamma_{2}}(s)\right\rangle=\frac{1}{\kappa_{\gamma_{3}}(s)} \frac{d}{d s}\left(\frac{\kappa_{\gamma_{1}}(s)}{\kappa_{\gamma_{2}}(s)} F(s)\right)
$$

and for each $i \in\{2,3, \ldots, n-3\}$, the $(i+1)$-th binormal component $\left\langle\gamma_{f}, B_{\gamma_{i+1}}\right\rangle$ of $\gamma_{f}$ is given by

$$
\left\langle\gamma_{f}(s), B \gamma_{i+1}(s)\right\rangle=\frac{1}{\kappa_{\gamma_{i+2}}(s)}\left[\kappa_{\gamma_{i+1}}(s)\left\langle\gamma_{f}(s), B_{\gamma_{i-1}}(s)\right\rangle+\left\langle\gamma_{f}(s), B_{\gamma_{i}}(s)\right\rangle\right] .
$$

Conversely, if $\gamma: I \longrightarrow \mathbb{E}^{n}$ is a unit-speed curve having nowhere vanishing $n-1$ curvatures $\kappa_{\gamma_{1}}, \kappa_{\gamma_{2}}, \ldots, \kappa_{\gamma_{n-1}}$, and $f: I \longrightarrow \mathbb{R}$ is a nowhere vanishing integrable function with at least $(n-2)$-times differentiable primitive function $F$ such that any one of the statements (1), (2), (3) or (4) is true, then $\gamma$ is an $f$-rectifying curve in $\mathbb{E}^{n}$.

Proof. First, for some nowhere vanishing integrable function $f: I \longrightarrow \mathbb{R}$ with at least $(n-2)$-times differentiable primitive function $F$, let $\gamma: I \longrightarrow \mathbb{E}^{n}$ be an $f$-rectifying curve in $\mathbb{E}^{n}$ having nowhere vanishing $n-1$ curvatures $\kappa_{\gamma_{1}}, \kappa_{\gamma_{2}}, \ldots, \kappa_{\gamma_{n-1}}$. Then for some differentiable functions $\lambda, \mu_{1}, \mu_{2}, \ldots, \mu_{n-2}: I \longrightarrow \mathbb{R}$, the $f$-position vector field $\gamma_{f}$ of $\gamma$ satisfies

$$
\gamma_{f}(s)=\lambda(s) T_{\gamma}(s)+\sum_{i=1}^{n-2} \mu_{i}(s) B_{\gamma_{i}}(s)
$$

Differentiating (4.1) and then applying the Frenet-Serret formulae (2.1), we obtain

$$
\begin{aligned}
f(s) T_{\gamma}(s)= & \lambda^{\prime}(s) T_{\gamma}(s)+\left(\lambda(s) \kappa_{\gamma_{1}}(s)-\mu_{1}(s) \kappa_{\gamma_{2}}(s)\right) N_{\gamma}(s)+\left(\mu_{1}^{\prime}(s)-\mu_{2}(s) \kappa_{\gamma_{3}}(s)\right) B_{\gamma_{1}}(s) \\
& +\sum_{i=2}^{n-3}\left(\mu_{i-1}(s) \kappa_{\gamma_{i+1}}(s)+\mu_{i}^{\prime}(s)-\mu_{i+1}(s) \kappa_{\gamma_{i+2}}(s)\right) B_{\gamma_{i}}(s)+\left(\mu_{n-3}(s) \kappa_{\gamma_{n-1}}(s)+\mu_{n-2}^{\prime}(s)\right) B \gamma_{n-2}(s)
\end{aligned}
$$

which gives the following set of relations

$$
\left\{\begin{aligned}
\lambda^{\prime}(s) & =f(s) \\
\lambda(s) \kappa_{\gamma_{1}}(s)-\mu_{1}(s) \kappa_{\gamma_{2}}(s) & =0 \\
\mu_{1}^{\prime}(s)-\mu_{2}(s) \kappa_{\gamma_{3}}(s) & =0 \\
\mu_{i-1}(s) \kappa_{\gamma_{i+1}}(s)+\mu_{i}^{\prime}(s)-\mu_{i+1}(s) \kappa_{\gamma_{i+2}}(s) & =0 \quad \text { for } i \in\{2,3, \ldots, n-3\}, \\
\mu_{n-3}(s) \kappa_{\gamma_{n-1}}(s)+\mu_{n-2}^{\prime}(s) & =0
\end{aligned}\right.
$$

From the first $n-1$ relations of (4.2), we find

$$
\left\{\begin{aligned}
\lambda(s) & =F(s) \\
\mu_{1}(s) & =\frac{\kappa_{\gamma_{1}}(s)}{\kappa_{\gamma_{2}}(s)} F(s) \\
\mu_{2}(s) & =\frac{1}{\kappa_{\gamma_{3}}(s)} \frac{d}{d s}\left(\frac{\kappa_{\gamma_{1}}(s)}{\kappa_{\gamma_{2}}(s)} F(s)\right), \\
\mu_{i+1}(s) & =\frac{1}{\kappa_{\gamma_{i+2}}(s)}\left[\mu_{i-1}(s) \kappa_{\gamma_{i+1}}(s)+\mu_{i}^{\prime}(s)\right] \text { for } i \in\{2,3, \ldots, n-3\} .
\end{aligned}\right.
$$


On the other hand, from the last $n-2$ relations of (4.2), we get

$$
\mu_{1}(s)\left(\mu_{1}^{\prime}(s)-\mu_{2}(s) \kappa_{\gamma_{3}}(s)\right)+\sum_{i=2}^{n-3} \mu_{i}(s)\left(\mu_{i-1}(s) \kappa_{\gamma_{i+1}}(s)+\mu_{i}^{\prime}(s)-\mu_{i+1}(s) \kappa_{\gamma_{i+2}}(s)\right)+\mu_{n-2}(s)\left(\mu_{n-2}^{\prime}(s)+\mu_{n-3}(s) \kappa_{\gamma_{n-1}}(s)\right)=0
$$

which reduces to

$$
\sum_{i=1}^{n-2} \mu_{i}(s) \mu_{i}^{\prime}(s)=0
$$

Integrating (4.4), we obtain

$$
\sum_{i=1}^{n-2} \mu_{i}^{2}(s)=c^{2}
$$

where $c$ is an arbitrary non-zero constant. Using (4.1), (4.3) and (4.5), the norm function $\rho=\left\|\gamma_{f}\right\|$ is given by

$$
\rho^{2}(s)=\left\|\gamma_{f}(s)\right\|^{2}=\left\langle\gamma_{f}(s), \gamma_{f}(s)\right\rangle=F^{2}(s)+\sum_{i=1}^{n-2} \mu_{i}^{2}(s)=F^{2}(s)+c^{2}
$$

This proves the statement (1). Again, using (4.1) and (4.3), the tangential component $\left\langle\gamma_{f}, T_{\gamma}\right\rangle$ of $\gamma_{f}$ is given by

$$
\left\langle\gamma_{f}(s), T_{\gamma}(s)\right\rangle=\lambda(s)=F(s) .
$$

This proves the statement (2). Now, for each $s \in I, \gamma_{f}(s)$ can be decomposed as

$$
\alpha_{f}(s)=v(s) T_{\gamma}(s)+\alpha_{f}^{N_{\gamma}}(s)
$$

for some differentiable function $v: I \longrightarrow \mathbb{R}$, where $\gamma_{f}^{N_{\gamma}}$ denotes the normal component of $\gamma_{f}$. Thus, in view of (4.1), $\gamma_{f}^{N_{\gamma}}$ is given by

$$
\gamma_{f}^{N_{\gamma}}(s)=\sum_{i=1}^{n-2} \mu_{i}(s) B_{\gamma_{i}}(s)
$$

Therefore, we have

$$
\left\|\gamma_{f}^{N_{\gamma}}(s)\right\|=\sqrt{\left\langle\gamma_{f}^{N_{\gamma}}(s), \gamma_{f}^{N_{\gamma}}(s)\right\rangle}=\sqrt{\sum_{i=1}^{n-2} \mu_{i}^{2}(s)} .
$$

Now, by using (4.5) in (4.6), we find $\left\|\gamma_{f}^{N_{\gamma}}(s)\right\|=c$. This proves the statement (3). Finally, using (4.1) and (4.3), the first binormal component $\left\langle\gamma_{f}, B_{\gamma_{1}}\right\rangle$ of $\gamma_{f}$ is given by

$$
\left\langle\gamma_{f}(s), B_{\gamma_{1}}(s)\right\rangle=\mu_{1}(s)=\frac{\kappa_{\gamma_{1}}(s)}{\kappa_{\gamma_{2}}(s)} F(s),
$$

the second binormal component $\left\langle\gamma_{f}, B_{\gamma_{2}}\right\rangle$ of $\gamma_{f}$ is given by

$$
\left\langle\gamma_{f}(s), B_{\gamma_{2}}(s)\right\rangle=\mu_{2}(s)=\frac{1}{\kappa_{\gamma_{3}}(s)} \frac{d}{d s}\left(\frac{\kappa_{\gamma_{1}}(s)}{\kappa_{\gamma_{2}}(s)} F(s)\right)
$$

and for each $i \in\{2,3, \ldots, n-3\}$, the $(i+1)$-th binormal component $\left\langle\gamma_{f}, B_{\gamma_{i+1}}\right\rangle$ of $\gamma_{f}$ is given by

$$
\left\langle\gamma_{f}(s), B_{\gamma_{i+1}}(s)\right\rangle=\mu_{i+1}(s)=\frac{1}{\kappa_{\gamma_{i+2}}(s)}\left[\kappa_{\gamma_{i+1}}(s)\left\langle\gamma_{f}(s), B_{\gamma_{i-1}}(s)\right\rangle+\left\langle\gamma_{f}(s), B_{\gamma_{i}}(s)\right\rangle\right] .
$$

Thus the statement (4) is proved.

Conversely, let $\gamma: I \longrightarrow \mathbb{E}^{n}$ be a unit-speed curve having nowhere vanishing $n-1$ curvatures $\kappa_{\gamma_{1}}, \kappa_{\gamma_{2}}, \ldots, \kappa_{\gamma_{n-1}}$, and $f: I \longrightarrow \mathbb{R}$ be a nowhere vanishing integrable function with at least $(n-2)$-times differentiable primitive function $F$ such that the statement (1) or the statement (2) is true. Then, in either case, we must have

$$
\left\langle\gamma_{f}(s), T_{\gamma}(s)\right\rangle=F(s)
$$

Differentiating (4.7) and then using the Frenet-Serret formulae (2.1), we finally obtain

$$
\left\langle\gamma_{f}(s), N_{\gamma}(s)\right\rangle=0
$$

This implies that $\gamma_{f}$ lies in the rectifying space of $\gamma$ and hence $\gamma$ is an $f$-rectifying curve in $\mathbb{E}^{n}$.

Next, we assume that the statement (3) is true. Then $\left\|\gamma_{f}^{N_{\gamma}}\right\|=$ a constant $=c$, say. Again, the normal component $\gamma_{f}^{N_{\gamma}}$ is given by

$$
\gamma_{f}(s)=F(s) T_{\gamma}(s)+\gamma_{f}^{N_{\gamma}}(s)
$$


and hence we have

$$
\left\langle\gamma_{f}(s), \gamma_{f}(s)\right\rangle=\left\langle\gamma_{f}(s), T_{\gamma}(s)\right\rangle^{2}+c^{2}
$$

Differentiating (4.8) and then applying the Frenet-Serret formulae (2.1), we obtain

$$
\left\langle\gamma_{f}(s), N_{\gamma}(s)\right\rangle=0
$$

This proves that $\gamma_{f}$ lies in the rectifying space of $\gamma$ and hence $\gamma$ is an $f$-rectifying curve in $\mathbb{E}^{n}$.

Finally, we assume that the statement (4) is true. Then the first binormal component and the second binormal component of $\gamma_{f}$ are respectively given by

$$
\begin{aligned}
\left\langle\gamma_{f}(s), B_{\gamma_{1}}(s)\right\rangle & =\frac{\kappa_{\gamma_{1}}(s)}{\kappa_{\gamma_{2}}(s)} F(s), \\
\left\langle\gamma_{f}(s), B_{\gamma_{2}}(s)\right\rangle & =\frac{1}{\kappa_{\gamma_{3}}(s)} \frac{d}{d s}\left(\frac{\kappa_{\gamma_{1}}(s)}{\kappa_{\gamma_{2}}(s)} F(s)\right) .
\end{aligned}
$$

Differentiating (4.9) and by using the Frenet-Serret formulae (2.1), we obtain

$$
-\kappa_{\gamma_{2}}(s)\left\langle\gamma_{f}(s), N_{\gamma}(s)\right\rangle+\kappa_{\gamma_{3}}(s)\left\langle\gamma_{f}(s), B_{\gamma_{2}}(s)\right\rangle=\frac{d}{d s}\left(\frac{\kappa_{\gamma_{1}}(s)}{\kappa_{\gamma_{2}}(s)} F(s)\right) .
$$

Combining (4.10) and (4.11), we find

$$
\left\langle\gamma_{f}(s), N_{\gamma}(s)\right\rangle=0
$$

Consequently, $\gamma_{f}$ lies in the rectifying space of $\gamma$ and hence $\gamma$ is an $f$-rectifying curve in $\mathbb{E}^{n}$.

\section{Classification of $f$-rectifying curves in $\mathbb{E}^{n}$}

In many papers (e.g., [3], [7], [8], [11] etc.), several interesting results were found primarily attempting towards the classification of rectifying curves which are mostly based on their parametrizations. In this section, we attempt for the same in $\mathbb{E}^{n}$ and this classification is totally based on the parametrizations of their $f$-position vector field.

Theorem 5.1. Let $\gamma: I \longrightarrow \mathbb{E}^{n}$ be a unit-speed curve (parametrized by arc-length s) having nowhere vanishing $n-1$ curvatures $\kappa_{\gamma_{1}}, \kappa_{\gamma_{2}}, \ldots, \kappa_{\gamma_{n-1}}$ and let $f: I \longrightarrow \mathbb{R}$ be a nowhere vanishing integrable function with at least $(n-2)$-times differentiable primitive function $F$. Then $\gamma$ is an $f$-rectifying curve in $\mathbb{E}^{n}$ if and only if, up to a parametrization, its $f$-position vector field $\gamma_{f}$ is given by

$$
\gamma_{f}(t)=c \sec \left(t+\arctan \left(\frac{F\left(s_{0}\right)}{c}\right)\right) \beta(t),
$$

where $c$ is a positive constant, $s_{0} \in I$ and $\beta: J \longrightarrow \mathbb{S}^{n-1}(1)$ is a unit-speed curve having $t: I \longrightarrow J$ as arc length function based at $s_{0}$.

Proof. First, for some nowhere vanishing integrable function $f: I \longrightarrow \mathbb{R}$ with at least $(n-2)$-times differentiable primitive function $F$, let $\gamma: I \longrightarrow \mathbb{E}^{n}$ be an $f$-rectifying curve having nowhere vanishing $n-1$ curvatures $\kappa_{\gamma_{1}}, \kappa_{\gamma_{2}}, \ldots, \kappa_{\gamma_{n-1}}$. Then by Theorem 4.1 , the norm function $\rho=\left\|\gamma_{f}\right\|$ is given by

$$
\rho(s)=\sqrt{F^{2}(s)+c^{2}},
$$

where we may choose $c$ as a positive constant. Now, we define a curve $\beta: I \longrightarrow \mathbb{E}^{n}$ by

$$
\beta(s):=\frac{1}{\rho(s)} \gamma_{f}(s)
$$

Then we find

$$
\langle\beta(s), \beta(s)\rangle=1 .
$$

Therefore, $\beta$ is a curve in the unit-sphere $\mathbb{S}^{n-1}(1)$. Differentiating (5.3), we get

$$
\left\langle\beta(s), \beta^{\prime}(s)\right\rangle=0 .
$$

Now, from (5.1) and (5.2), we obtain

$$
\gamma_{f}(s)=\beta(s) \sqrt{F^{2}(s)+c^{2}} .
$$

Again, differentiating (5.5), we obtain

$$
f(s) T_{\gamma}(s)=\beta^{\prime}(s) \sqrt{F^{2}(s)+c^{2}}+\frac{\beta(s) f(s) F(s)}{\sqrt{F^{2}(s)+c^{2}}} .
$$


Using (5.3), (5.4) and (5.6), we obtain

Therefore, we get

$$
\left\langle\beta^{\prime}(s), \beta^{\prime}(s)\right\rangle=\frac{c^{2} f^{2}(s)}{\left(F^{2}(s)+c^{2}\right)^{2}}
$$

$$
\left\|\beta^{\prime}(s)\right\|=\sqrt{\left\langle\beta^{\prime}(s), \beta^{\prime}(s)\right\rangle}=\frac{c f(s)}{F^{2}(s)+c^{2}} .
$$

Now, for some $s_{0} \in I$, let $t: I \longrightarrow J$ be arc-length parameter of $\beta$ given by

$$
t=\int_{s_{0}}^{s}\left\|y^{\prime}(u)\right\| d u
$$

Then we have

$$
\begin{aligned}
t & =\int_{s_{0}}^{s} \frac{c f(u)}{F^{2}(u)+c^{2}} d u \\
\Longrightarrow \quad t & =\arctan \left(\frac{F(s)}{c}\right)-\arctan \left(\frac{F\left(s_{0}\right)}{c}\right) \\
\Longrightarrow \quad s & =F^{-1}\left(c \tan \left(t+\arctan \left(\frac{F\left(s_{0}\right)}{c}\right)\right)\right) .
\end{aligned}
$$

Substituting (5.10) in (5.5), we obtain the $f$-position vector field of $\gamma$ as follows:

$$
\gamma_{f}(t)=c \sec \left(t+\arctan \left(\frac{F\left(s_{0}\right)}{c}\right)\right) \beta(t) .
$$

Conversely, let $\gamma$ be a unit-speed curve in $\mathbb{E}^{n}$ such that, up to a parametrization, its $f$-position vector field $\gamma_{f}$ is defined by

$$
\gamma_{f}(t):=c \sec \left(t+\arctan \left(\frac{F\left(s_{0}\right)}{c}\right)\right) \beta(t)
$$

where $c$ is a positive constant and $\beta: J \longrightarrow \mathbb{S}^{n-1}(1)$ is a unit-speed curve having $t: I \longrightarrow J$ as arc length function based at $s_{0}$. Differentiating (5.11), we obtain

$$
\gamma_{f}^{\prime}(t)=c \sec \left(t+\arctan \left(\frac{F\left(s_{0}\right)}{c}\right)\right)\left[\tan \left(t+\arctan \left(\frac{F\left(s_{0}\right)}{c}\right)\right) \beta(t)+1\right] \beta^{\prime}(t) .
$$

Since $\beta$ is a unit-speed curve in the unit-sphere $\mathbb{S}^{n-1}(1)$, we have $\left\langle\beta^{\prime}(t), \beta^{\prime}(t)\right\rangle=1,\langle\beta(t), \beta(t)\rangle=1$ and consequently $\left\langle\beta(t), \beta^{\prime}(t)\right\rangle=0$. Therefore, from (5.11) and (5.12), we have

$$
\begin{aligned}
\left\langle\gamma_{f}(t), \gamma_{f}(t)\right\rangle & =c^{2} \sec ^{2}\left(t+\arctan \left(\frac{F\left(s_{0}\right)}{c}\right)\right) \\
\left\langle\gamma_{f}(t), \gamma_{f}^{\prime}(t)\right\rangle & =c^{2} \sec ^{2}\left(t+\arctan \left(\frac{F\left(s_{0}\right)}{c}\right)\right) \tan \left(t+\arctan \left(\frac{F\left(s_{0}\right)}{c}\right)\right) \\
\left\langle\gamma_{f}^{\prime}(t), \gamma_{f}^{\prime}(t)\right\rangle & =c^{2} \sec ^{4}\left(t+\arctan \left(\frac{F\left(s_{0}\right)}{c}\right)\right) .
\end{aligned}
$$

Now, if we put

$$
t=\arctan \left(\frac{F(s)}{c}\right)-\arctan \left(\frac{F\left(s_{0}\right)}{c}\right)
$$

then $s$ becomes arc length parameter of $\gamma$ and equations (5.13), (5.14), (5.15) reduce to

$$
\begin{aligned}
\left\langle\gamma_{f}(s), \gamma_{f}(s)\right\rangle & =c^{2} \sec ^{2}\left(\frac{F(s)}{c}\right), \\
\left\langle\gamma_{f}(s), \gamma_{f}^{\prime}(s)\right\rangle & =c^{2} \sec ^{2}\left(\frac{F(s)}{c}\right) \tan \left(\frac{F(s)}{c}\right), \\
\left\langle\gamma_{f}^{\prime}(s), \gamma_{f}^{\prime}(s)\right\rangle & =c^{2} \sec ^{4}\left(\frac{F(s)}{c}\right) .
\end{aligned}
$$

Again, the normal component $\gamma_{f}^{N_{\gamma}}$ of $\gamma_{f}$ is given by

$$
\left\langle\gamma_{f}^{N_{\gamma}}(s), \gamma_{f}^{N_{\gamma}}(s)\right\rangle=\left\langle\gamma_{f}(s), \gamma_{f}(s)\right\rangle-\frac{\left\langle\gamma_{f}(s), \gamma_{f}^{\prime}(s)\right\rangle^{2}}{\left\langle\gamma_{f}^{\prime}(s), \gamma_{f}^{\prime}(s)\right\rangle} .
$$

Then substituting (5.16), (5.17)) and (5.18) in the previous equation, we obtain

$$
\left\langle\gamma_{f}^{N_{\gamma}}(s), \gamma_{f}^{N_{\gamma}}(s)\right\rangle=\left\|\gamma_{f}^{N_{\gamma}}(s)\right\|^{2}=c^{2}
$$

This implies that the normal component $\gamma_{f}^{N_{\gamma}}$ of $\gamma_{f}$ has a constant length. Also, the norm function $\rho=\left\|\gamma_{f}\right\|$ is given by

$$
\rho(s)=\sqrt{\left\langle\gamma_{f}(s), \gamma_{f}(s)\right\rangle}=c \sec \left(\frac{F(s)}{c}\right)
$$

and it is non-constant. Therefore, by applying the Theorem 4.1, we conclude that $\gamma$ is an $f$-rectifying curve in $\mathbb{E}^{n}$. 


\section{Conclusion}

It goes without saying that $f$-rectifying curves in Euclidean spaces are a sort of generalizations of rectifying curves therein. In this paper, we presented a study on $f$-rectifying curves in Euclidean $n$-space $\mathbb{E}^{n}$. Predominantly, we explored two main theorems demonstrating some necessary and sufficient conditions for a regular curve to be an $f$-rectifying curve in $\mathbb{E}^{n}$. The first theorem portrays some geometric characterizations of $f$-rectifying curves in $\mathbb{E}^{n}$ in connection with norm functions, tangential, normal and $n-2$ binormal components of their $f$-position vector field. Whereas the second theorem classifies such curves based on parametrization of their $f$-position vector field. Moreover, it yields an important characterization: namely, the $f$-position vector field of an $f$-rectifying curve in $\mathbb{E}^{n}$ is a dilation of a unit-speed curve in the unit $(n-1)$-sphere $\mathbb{S}^{n-1}(1)$ with dilation factor $c \sec \left(t+\arctan \left(\frac{F\left(s_{0}\right)}{c}\right)\right)$ for some constants $c>0$ and $s_{0}$. Extensions of such study to other ambient spaces may be considered as problems of interest.

\section{Acknowledgement}

We would like to express our sincere thanks to the anonymous referees for their time dedicated to this paper and for their invaluable comments and suggestions which definitely helped to improve this paper.

\section{References}

[1] M.P. Do Carmo, Differential Geometry of Curves and Surfaces: Revised and Updated Second Edition, Dover Publications Inc., New York, 2016.

[2] A.N. Pressley, Elementary Differential Geometry, Second Edition, Springer, London, 2010.

[3] B.Y. Chen, When does the position vector of a space curve always lie in its rectifying plane? Amer. Math. Monthly, 110(2) (2003), 147-152.

[4] B.Y. Chen, F. Dillen, Rectifying curves as centrodes and extremal curves, Bull. Inst. Math. Acad. Sinica, 33(2) (2005), 77-90.

[5] B.Y. Chen, Rectifying curves and geodesics on a cone in the Euclidean 3-space, Tamkang J. Math., 48(2) (2017), 209-214.

[6] S. Deshmukh, B.Y. Chen, S. Alshamari, On rectifying curves in Euclidean 3-space, Turk. J. Math., 42(2) (2018), 609-620.

[7] K. İlarslan, E. Nesovic, Some characterizations of rectifying curves in the Euclidean space $\mathbf{E}^{4}$, Turk. J. Math., 32(1) (2008), 21-30.

[8] S. Cambie, W. Goemans, I. Van den Bussche, Rectifying curves in the n-dimensional Euclidean space, Turk. J. Math., 40(1) (2016), 210-223.

[9] P. Lucas, J.A. Ortega-Yaguies, Rectifying curves in the three-dimensional sphere, J. Math. Anal. Appl., 421(2) (2015), 1855-1868.

[10] P. Lucas, J.A. Ortega-Yagües, Rectifying curves in the three-dimensional hyperbolic space, Medit. J. Math., 13(4) (2016), $2199-2214$.

[11] K. Ilarslan, E. Nešović, T.M. Petrović, Some characterization of rectifying curves in the Minkowski 3-Space, Novi Sad J. Math., 33(2) (2003), 23-32.

[12] K. Ilarslan, E. Nešović, On rectifying curves as centrodes and extremal curves in the Minkowski 3-Space, Novi Sad J. Math., 37(1) (2007), 53-64.

[13] K. Ilarslan, E. Nešović, Some characterizations of null, pseudo null and partially null rectifying curves in Minkowski space-time, Taiwanese J. Math., 12(5) (2008), 1035-1044.

[14] T.A. Ali, M. Onder, Some characterizations of space-like rectifying curves in the Minkowski space-time, Glob. J. Sci. Fron. Res. Math. Des. Sci., 12(1) (2012), 57-63.

[15] K. Ilarslan, E. Nešović, Some relations between normal and rectifying curves in Minkowski space-time, Inter. Elec. J. Geom., 7(1) (2014), 26-35.

[16] F. Hathout, A new class of curves generalizing helix and rectifying curves, arXiv: Diff. Geom., (2018).

[17] Z. Iqbal, J. Sengupta, Non-null (spacelike or timelike) f-rectifying curves in the Minkowski 3-space $\mathbb{E}_{1}^{3}$, Eurasian Bul. Math., 3(1) (2020), 38-55.

[18] Z. Iqbal, J. Sengupta, Null (lightlike) f-rectifying curves in the Minkowski 3-space $\mathbb{E}_{1}^{3}$, Fundam. J. Math. Appl., 3(1) (2020), 8-16.

[19] Z. Iqbal, J. Sengupta, Differential geometric aspects of lightlike f-rectifying curves in Minkowski space-time, Diff. Geom. - Dyn. Syst., 22 (2020), $113-129$.

[20] Z. Iqbal, J. Sengupta, On f-Rectifying Curves in the Euclidean 4-Space, Acta Univ. Sapientiae Math., 13(1) (2021), $192-208$.

[21] H. Gluck, Higher curvatures of curves in Euclidean space, Amer. Math. Monthly, 73(7) (1966), 699-704.

[22] H. Gluck, Higher curvatures of curves in Euclidean space II, Amer. Math. Monthly, 74(9) (1967), 1049-1056. 